University of Windsor

Scholarship at UWindsor

\title{
Variational energies and the Fermi contact term for the low-lying states of lithium: Basis-set completeness
}

L. M. Lang

Z. C. Yan

H. X. Qiao

Gordon W. F. Drake

University of Windsor

Follow this and additional works at: https://scholar.uwindsor.ca/physicspub

Part of the Physics Commons

\section{Recommended Citation}

Lang, L. M.; Yan, Z. C.; Qiao, H. X.; and Drake, Gordon W. F.. (2012). Variational energies and the Fermi contact term for the low-lying states of lithium: Basis-set completeness. Physical Review A - Atomic, Molecular, and Optical Physics, 85 (5), 52513-1-52513-9.

https://scholar.uwindsor.ca/physicspub/116

This Article is brought to you for free and open access by the Department of Physics at Scholarship at UWindsor. It has been accepted for inclusion in Physics Publications by an authorized administrator of Scholarship at UWindsor. For more information, please contact scholarship@uwindsor.ca. 


\title{
Variational energies and the Fermi contact term for the low-lying states of lithium: Basis-set completeness
}

\author{
L. M. Wang, ${ }^{1,2}$ Z.-C. Yan, ${ }^{1}$ H. X. Qiao, ${ }^{2}$ and G. W. F. Drake ${ }^{3}$ \\ ${ }^{1}$ Department of Physics, University of New Brunswick, Fredericton, New Brunswick, Canada E3B 5 A3 \\ ${ }^{2}$ Department of Physics, Wuhan University, Wuhan 430072, China \\ ${ }^{3}$ Department of Physics, University of Windsor, Windsor, Ontario, Canada N9B 3P4
}

(Received 11 March 2012; published 24 May 2012)

\begin{abstract}
Nonrelativistic energies for the low-lying states of lithium are calculated using the variational method in Hylleraas coordinates. Variational eigenvalues for the infinite nuclear mass case with up to 34020 terms are $-7.478060323910147(1)$ a.u. for $1 s^{2} 2 s^{2} S,-7.35409842144437(1)$ a.u. for $1 s^{2} 3 s^{2} S,-7.31853084599891(1)$ a.u. for $1 s^{2} 4 s^{2} S,-7.41015653265241(4)$ a.u. for $1 s^{2} 2 p^{2} P$, and $-7.335523543524688(3)$ a.u. for $1 s^{2} 3 d^{2} D$. The selection of the minimum set of angular momentum configurations is discussed, with the $2 P$ and $3 D$ states as examples to demonstrate the impact of various configurations on the variational energies. It is shown by numerical example that the second spin function (i.e., coupled to form a triplet intermediate state) has no significant effect on either the variational energies or the spin-dependent Fermi contact term. Results of greatly improved accuracy for the Fermi contact term are presented for all the states considered.
\end{abstract}

DOI: 10.1103/PhysRevA.85.052513

PACS number(s): 31.15.ac, 31.15.aj

\section{INTRODUCTION}

The ultimate aim of theoretical atomic spectroscopy is to match or exceed the state of the art for accuracy in spectroscopic measurements. A comparison with the measurements then yields tests of fundamental theory, or new values for physical parameters such as fundamental constants or properties of the nucleus. It is currently not possible to achieve this goal of accuracy for many-electron atoms, even in the nonrelativistic limit, but it is possible for hydrogen, helium, and more recently also for lithium.

The most accurate results are achieved for two- and three-electron atoms by using the Rayleigh-Ritz variational method in Hylleraas coordinates. Though many difficulties still remain, significant advances have been achieved in the past two decades [1-9]. Especially in the past five years, the rate of progress has accelerated. For example, the ground-state energy of lithium has been calculated to 14 digit accuracy $[8,9]$ and similarly for the $3 S$ state [9]. Needless to say, these advances are closely correlated with the progress of computer technology. Basis sets containing as many as 27000 terms were used in our recent work [9]. At this level, parallel processing becomes a necessity.

Other competitive methods are also under development. Sims and Hagstrom [10] used the Hy-CI (Hylleraasconfiguration-interaction) method to calculate the ground-state energy of lithium to be -7.478060323452 a.u. with 16764 basis functions. Stanke et al. [11] used a method based on explicitly correlated Gaussian basis functions [12] to calculate the ground-state energy of lithium to -7.47806032381 a.u. with 10000 terms. These two results are both very accurate, but still several orders of magnitude less than the most accurate one obtained by using pure Hylleraas coordinates. This verifies that a variational method based on Hylleraas coordinates is still the most accurate method for solving the Schrödinger equation for two- and three-electron atoms.

One of the problems exhibited by Hylleraas basis sets is that, as the basis set is enlarged, it tends to develop a near linear dependence and numerical instability. To avoid this problem, Yan and Drake [2] (see also McKenzie and Drake [1]) introduced Hylleraas basis sets partitioned into multiple sectors with different distance scales that are individually optimized for each sector. These basis sets have been verified to be very efficient and stable in practical calculations [2-6]. In our recent paper [9], we made a slight modification to this method of choosing the basis sets and found that the new method is more efficient than the old one, and is still stable. The modifications involved subdividing one sector and truncating the terms in another, but this remains a matter of trial and error with few general rules to guide one's intuition. In particular, rules that appear to be true for small basis sets may no longer hold for large basis sets.

One issue that has not been adequately explored is the role of the second spin function. As well known, a spin-doublet state of lithium can be formed by coupling a third electron to either an intermediate singlet pair, or an intermediate triplet pair. Usually just the former is used in calculations. The impact of not including the latter triplet has been studied by many authors [13-17]. Their common conclusion is that including the triplet spin coupling explicitly will not enhance the convergence of the energy significantly, but it will strongly affect the expectation values of other operators which are spin dependent, such as the Fermi contact term. In this paper, we will give an argument to show that the triplet spin wave function is indispensable for the correct wave function of the system, though the energy is not very sensitive to it.

A further problem concerns the selection of angular momentum configurations for non- $S$ states. The problem is well studied for two-electron systems in Hylleraas coordinates by Breit [18], Schwartz [19], and Drake [20], but for the three-electron case little had been published before the work of Harris [21] in 2005. In the present paper, we reinvestigate this problem and take the $2 P$ and $3 D$ states of lithium as examples to demonstrate the impact of various angular momentum configurations on the energy levels. 
The rest of the paper is organized as follows. Section II discusses the overall form of the variational wave function, and especially the partitioning of the radial part into sectors with individually optimized distance scales. Section III presents a general discussion of the various angular couplings that are required for completeness of the variational basis set, and Sec. IV presents results for the $2 P$ and $3 D$ states to illustrate the role played by different angular momentum couplings. Section V studies in detail the effect of the second spin function on both the energies and the spin-dependent Fermi contact term. Finally, Sec. VI contains a brief summary of the results and their significance.

\section{CONSTRUCTION OF BASIS SETS}

For the sake of completeness, we briefly describe the structure of variational basis sets, based on our early work [2]. Further modifications to obtain better convergence of energy eigenvalues will be discussed in Secs. III and V.

The variational wave function is a linear combination of terms of the form (in atomic units throughout)

$$
\psi=\mathcal{A}\left(\phi\left(\mathbf{r}_{1}, \mathbf{r}_{2}, \mathbf{r}_{3}\right)\right)
$$

where

$$
\begin{aligned}
\phi\left(\mathbf{r}_{1}, \mathbf{r}_{2}, \mathbf{r}_{3}\right)= & r_{1}^{j_{1}} r_{2}^{j_{2}} r_{3}^{j_{3}} r_{12}^{j_{12}} r_{23}^{j_{23}} r_{31}^{j_{31}} e^{-\alpha r_{1}-\beta r_{2}-\gamma r_{3}} \\
& \times \mathcal{Y}_{\left(\ell_{1} \ell_{2}\right) \ell_{12}, \ell_{3}}^{L M}\left(\mathbf{r}_{1}, \mathbf{r}_{2}, \mathbf{r}_{3}\right) \chi_{1}
\end{aligned}
$$

and

$$
\begin{aligned}
& \mathcal{Y}_{\left(\ell_{1} \ell_{2}\right) \ell_{12}, \ell_{3}}^{L M}\left(\mathbf{r}_{1}, \mathbf{r}_{2}, \mathbf{r}_{3}\right) \\
& =r_{1}^{\ell_{1}} r_{2}^{\ell_{2}} r_{3}^{\ell_{3}} \sum_{m_{i}}\left\langle\ell_{1} m_{1} ; \ell_{2} m_{2} \mid \ell_{1} \ell_{2} ; \ell_{12} m_{12}\right\rangle \\
& \quad \times\left\langle\ell_{12} m_{12} ; \ell_{3} m_{3} \mid \ell_{12} \ell_{3} ; L M\right\rangle \\
& \quad \times Y_{\ell_{1} m_{1}}\left(\mathbf{r}_{1}\right) Y_{\ell_{2} m_{2}}\left(\mathbf{r}_{2}\right) Y_{\ell_{3} m_{3}}\left(\mathbf{r}_{3}\right)
\end{aligned}
$$

is the vector-coupled product of spherical harmonics for the three electrons to form a state of total angular momentum $L$ and $z$ component $M$,

$$
\chi_{1}=\alpha(1) \beta(2) \alpha(3)-\beta(1) \alpha(2) \alpha(3)
$$

is the spin function with the total spin $1 / 2$, and

$$
\mathcal{A}=(1)-(12)-(13)-(23)+(123)+(132)
$$

is the three-particle antisymmetrizer. The quantities $r_{i}, i=$ $1,2,3$ are the radial coordinates for the three electrons, and the $r_{i j}=\left|\mathbf{r}_{i}-\mathbf{r}_{j}\right|$ are the interparticle coordinates. The angular momenta $\ell_{i}$ are chosen according to

$$
\begin{aligned}
& \left(\ell_{1}, \ell_{2}, \ell_{3}\right)=(0,0,0)_{A} \quad \text { for } S \text { states, } \\
& \left(\ell_{1}, \ell_{2}, \ell_{3}\right)=(0,0,1)_{A},(0,1,0)_{B} \quad \text { for } P \text { states, } \\
& \left(\ell_{1}, \ell_{2}, \ell_{3}\right)=(0,0,2)_{A},(0,1,1)_{B} \quad \text { for } D \text { states. }
\end{aligned}
$$

As described previously [2], all terms in Eq. (2) are nominally included such that

$$
j_{1}+j_{2}+j_{3}+j_{12}+j_{23}+j_{31} \leqslant \Omega
$$

and the convergence of the eigenvalues is studied as $\Omega$ is progressively increased. However, terms that may potentially cause near linear dependence are omitted. For example, if $\ell_{1}=\ell_{2}$ and $\alpha \approx \beta$, then terms with $j_{1}>j_{2}$ are omitted, as well as terms with $j_{1}=j_{2}$ when $j_{23}>j_{31}$. The presence of the near-linear dependency problem in a basis set may be detected by diagonalizing the positive-definite overlap matrix to check for abnormally small or negative eigenvalues. Furthermore, the first sector $(0,0, L)_{A}$ is further divided into five subsectors according to correlations among the three electrons

$$
\begin{array}{llll}
\text { sector } 1: & \text { all } j_{12}, & j_{23}=0, & j_{31}=0, \\
\text { sector } 2: & \text { all } j_{12}, & j_{23}=0, & j_{31} \neq 0, \\
\text { sector 3: } & \text { all } j_{12}, & j_{23} \neq 0, & j_{31}=0, \\
\text { sector } 4: & j_{12}=0, & j_{23} \neq 0, & j_{31} \neq 0, \\
\text { sector 5: } & j_{12} \neq 0, & j_{23} \neq 0, & j_{31} \neq 0 .
\end{array}
$$

Thus the basis sets contain five sectors for $S$ states and six sectors for $P$ and $D$ states including $(0,1,0)_{B}$ or $(0,1,1)_{B}$. The size of each sector is separately determined by assigning to each an $\Omega_{i}$ according to

$$
\begin{aligned}
\left\{\Omega_{1}, \Omega_{2}, \Omega_{3}, \Omega_{4}, \Omega_{5}\right\} & =\{\Omega, \Omega, \Omega, \Omega, \Omega\}, \quad L=0 \\
\left\{\Omega_{1}, \Omega_{2}, \Omega_{3}, \Omega_{4}, \Omega_{5}, \Omega_{6}\right\} & =\{\Omega, \Omega, \Omega, \Omega, \Omega, \Omega-2\}, \quad L=1,2
\end{aligned}
$$

\section{COMPLETENESS OF HYLLERAAS-TYPE BASIS SETS}

For the $S$ states of two-electron atomic systems, the completeness of a Hylleraas-type basis set has been proved rigorously by Klahn and Bingel [22]. Their detailed proof is lengthy, but the problem can be simply understood from the viewpoint of configuration interaction involving a multipole expansion. By definition $r_{12}^{2 n}$ can be written in the form

$$
r_{12}^{2 n}=\left[r_{1}^{2}+r_{2}^{2}-2 \mathbf{r}_{1} \cdot \mathbf{r}_{2}\right]^{n}
$$

and $\mathbf{r}_{1} \cdot \mathbf{r}_{2}=r_{1} r_{2} \cos \theta_{12}$, where $\theta_{12}$ is the angle between the vectors $\mathbf{r}_{1}$ and $\mathbf{r}_{2}$. Thus the right-hand side of Eq. (9) contains terms up to $\cos ^{n} \theta_{12}$, which can be reexpressed as finite linear combinations of Legendre polynomials up to $P_{n}\left(\cos \theta_{12}\right)$. Each Legendre polynomial can in turn be expanded into multipoles according to the spherical harmonic addition theorem

$$
P_{q}\left(\cos \theta_{12}\right)=\frac{4 \pi}{2 q+1} \sum_{m=-q}^{q}(-1)^{m} Y_{q-m}\left(\mathbf{r}_{1}\right) Y_{q m}\left(\mathbf{r}_{2}\right) .
$$

Since $\cos \theta_{12}$ is rotationally invariant, so also is the sum over spherical harmonics on the right-hand side. Thus each term $P_{q}\left(\cos \theta_{12}\right)$ is equivalent to the coupling of two electrons of angular momentum $q$ to form an $S$ state; and so the even powers of $r_{12}$ alone, when multiplied into the uncorrelated wave function with independent variational parameters, are equivalent to the partial wave expansion of a configuration interaction (CI) wave function for an $S$ state, and a CI wave function is known to be complete when all partial waves are included. The angular momentum couplings generated are thus of the form $\left(s s^{\prime}\right){ }^{1} S+\left(p p^{\prime}\right){ }^{1} S+\left(d d^{\prime}\right){ }^{1} S+\cdots$, with independent variational coefficients for each term. The odd powers of $r_{12}$ greatly accelerate the rate of convergence because they are suited to a representation of the electron cusp condition along the line $r_{1}=r_{2}$. For an example, see Ref. [23]. The problem of completeness for the three-electron case can be understood in a similar way, as discussed further below.

For the non- $S$ states of two-electron atomic systems, the problem of the completeness of Hylleraas coordinates has been 
studied by Breit [18], Schwartz [19], and Drake [20]. The common conclusion of these authors is that, because $r_{12}^{n}$ is included explicitly in the Hylleraas-type basis functions, the addition of at most $[L / 2]$ angular momentum configurations is sufficient to achieve completeness in the limit as the basis set is enlarged, where $[L / 2]$ denotes "greatest integer in." A detailed rule for selecting these additional angular momentum configurations has been given by Drake [20]. For a singly excited state of angular momentum $L$, the minimum $\left(\ell_{1}, \ell_{2}\right)$ configurations needed in the basis set are those with $\ell_{1}+\ell_{2}=$ $L$, and $\ell_{1} \leqslant \ell_{2}$. As a simple example, for $L=2$ the essential angular couplings are of the form $s d^{1} D$ and $p p^{\prime}{ }^{1} D$, and similarly for the triplets.

For the non- $S$ states of three-electron atomic systems, the problem of the completeness of Hylleraas coordinates was recently discussed by Harris [21], building on the earlier work of Schwartz [19] for the two-electron case. We first summarize the main points of Schwartz and Harris and then give the necessary angular momentum configurations for $2 P$ and $3 D$ states of lithium.

The basic form of the Hylleraas-type basis functions for three-electron systems is described by Eq. (2) and the angular part of the functions is described by Eq. (3). As for the two-electron case, we first consider the additional angular functions that are generated when the basic function in Eq. (3) is multiplied by powers of the $r_{i j}$ coordinates. It follows from the triangular rule for angular momentum coupling and parity selection rules that

$$
\cos \theta Y_{\ell, m}(\theta, \phi)=A_{\ell, m}^{\ell-1} Y_{\ell-1, m}(\theta, \phi)+A_{\ell, m}^{\ell+1} Y_{\ell+1, m}(\theta, \phi) .
$$

The only nonvanishing values for the coefficients $A_{\ell, m}^{\ell^{\prime}}$ are

$$
\begin{aligned}
A_{\ell, m}^{\ell^{\prime}}= & (-1)^{m}\left[(2 \ell+1)\left(2 \ell^{\prime}+1\right)\right]^{1 / 2} \\
& \times\left(\begin{array}{ccc}
1 & \ell & \ell^{\prime} \\
0 & m & -m
\end{array}\right)\left(\begin{array}{ccc}
1 & \ell & \ell^{\prime} \\
0 & 0 & 0
\end{array}\right)
\end{aligned}
$$

expressed in terms of $3 j$ symbols [24] with $\ell^{\prime}=\ell \pm 1$. The general form for $\hat{\mathbf{r}}_{1} \cdot \hat{\mathbf{r}}_{2}$ acting on the initial $\mathcal{Y}_{\left(\ell_{1} \ell_{2}\right) \ell_{12}, \ell_{3}}^{L M}$ is thus

$$
\begin{aligned}
\left(\hat{\mathbf{r}}_{1} \cdot \hat{\mathbf{r}}_{2}\right) & \mathcal{Y}_{\left(\ell_{1} \ell_{2}\right) \ell_{12}, \ell_{3}}^{L M} \\
= & C_{1} \mathcal{Y}_{\left(\ell_{1}-1, \ell_{2}-1\right) \ell_{12}, \ell_{3}}^{L M}+C_{2} \mathcal{Y}_{\left(\ell_{1}-1, \ell_{2}+1\right) \ell_{12}, \ell_{3}}^{L M} \\
& +C_{3} \mathcal{Y}_{\left(\ell_{1}+1, \ell_{2}-1\right) \ell_{12}, \ell_{3}}^{L M}+C_{4} \mathcal{Y}_{\left(\ell_{1}+1, \ell_{2}+1\right) \ell_{12}, \ell_{3}}^{L M},
\end{aligned}
$$

where the $C_{i}$ are constants, and all $\ell_{i} \pm 1$ are non-negative. Thus successive powers of $\hat{\mathbf{r}}_{1} \cdot \hat{\mathbf{r}}_{2}$ generate a wide variety of configurations $\left(\left(\ell_{1}^{\prime}, \ell_{2}^{\prime}\right) \ell_{12}, \ell_{3} ; L M\right)$, but they are not all linearly independent because of the fixed $C_{i}$ coefficients in Eq. (13). Similarly powers of $\hat{\mathbf{r}}_{2} \cdot \hat{\mathbf{r}}_{3}$ generate configurations of the form $\left(\left(\ell_{1}, \ell_{2}^{\prime}\right) \ell_{12}^{\prime}, \ell_{3}^{\prime} ; L M\right)$, but again they are not all linearly independent. For a state with definite total angular momentum $L$, only those configurations satisfying the following conditions need be included explicitly in Hylleraas bases [21]:

$$
\begin{gathered}
\ell_{1}+\ell_{2}+\ell_{3}=L \quad \text { for parity }(-1)^{L}, \\
\ell_{1}+\ell_{2}+\ell_{3}=L+1 \text { for parity }(-1)^{(L+1)} .
\end{gathered}
$$

Another problem that should be emphasized is that the coupling schemes of angular momenta for three-body systems are not unique [24]. The three distinct coupling schemes are (in
TABLE I. Angular momentum configurations for $P$ states (odd parity) of lithium.

\begin{tabular}{lcccccc}
\hline \hline No. & $\ell_{1}$ & $\ell_{2}$ & $\ell_{12}$ & $\ell_{3}$ & $L$ & $M$ \\
\hline 1 & 0 & 0 & 0 & 1 & 1 & 0 \\
2 & 0 & 1 & 1 & 0 & 1 & 0 \\
3 & 1 & 0 & 1 & 0 & 1 & 0 \\
\hline \hline
\end{tabular}

an obvious notation) $\left|\left(\ell_{1}, \ell_{2}\right) \ell_{12} \ell_{3} ; L M\right\rangle,\left|\ell_{1}\left(\ell_{2}, \ell_{3}\right) \ell_{23} ; L M\right\rangle$, and $\left|\left(\ell_{1}, \ell_{3}\right) \ell_{13} \ell_{2} ; L M\right\rangle$. In general, there are multiple possible values for the intermediate angular momenta $\ell_{12}, \ell_{23}$, and $\ell_{13}$. In fact, these three-coupling schemes should be physically equivalent. This is ensured by the possibility of unitary transformations among these three schemes. For example, there is a relationship between the state vectors of the first and second according to the recoupling transformation

$$
\begin{aligned}
& \left|\ell_{1}\left(\ell_{2}, \ell_{3}\right) \ell_{23} ; L M\right\rangle \\
& =(-1)^{\ell_{1}+\ell_{2}+\ell_{3}+L} \sum_{\ell_{12}^{\prime}} \sqrt{\left(2 \ell_{12}^{\prime}+1\right)\left(2 \ell_{23}+1\right)} \\
& \quad \times\left\{\begin{array}{lll}
\ell_{1} & \ell_{2} & \ell_{12}^{\prime} \\
\ell_{3} & L & \ell_{23}
\end{array}\right\}\left|\left(\ell_{1}, \ell_{2}\right) \ell_{12}^{\prime} \ell_{3} ; L M\right\rangle,
\end{aligned}
$$

expressed in terms of the standard $6 j$ symbols [24]. It can be seen from the above equation that the transformation from the first to the second scheme can be completed only if all possible values of $\ell_{12}$ are included in the basis set. So, the values of the intermediate angular momenta $\left(\ell_{12}\right.$, or $\ell_{23}$, or $\left.\ell_{13}\right)$ are important.

\section{RESULTS FOR THE $2 P$ AND $3 D$ STATES}

Following the discussions of the preceding section, we list the necessary angular momentum configurations for $2 P$ and $3 D$ states of lithium in Tables I and II, respectively. For the $2 P$ state, there are three configurations in total. As pointed out by Yan and Drake [2], the energy of the $1 s^{2} 2 p^{2} P$ state would converge to a wrong value if only the first configuration in Table I were used. This is evidence of the incompleteness of the basis set. In Table III, we list the energies of the $2 P$ state calculated using two configurations and three configurations. The results calculated using two configurations are quoted from our recent paper [9]. The variational basis set for the calculations using three configurations is constructed as follows: the whole basis set is divided into seven sectors, and the radial parts of the first four sectors are generated according to the basic manner described previously, namely, formula (8).

TABLE II. Angular momentum configurations for $D$ states (even parity) of lithium.

\begin{tabular}{lcccccc}
\hline \hline No. & $\ell_{1}$ & $\ell_{2}$ & $\ell_{12}$ & $\ell_{3}$ & $L$ & $M$ \\
\hline 1 & 0 & 0 & 0 & 2 & 2 & 0 \\
2 & 0 & 1 & 1 & 1 & 2 & 0 \\
3 & 0 & 2 & 2 & 0 & 2 & 0 \\
4 & 1 & 0 & 1 & 1 & 2 & 0 \\
5 & 2 & 0 & 2 & 0 & 2 & 0 \\
6 & 1 & 1 & 2 & 0 & 2 & 0 \\
\hline \hline
\end{tabular}


TABLE III. Convergence study for the nonrelativistic energy of lithium in the $1 s^{2} 2 p^{2} P$ state, calculated using two and three angular momentum configurations, respectively, and with the second spin function $\chi_{2}$. Units are atomic units, and $\Omega=\infty$ denotes the extrapolated value.

\begin{tabular}{|c|c|c|c|}
\hline$\Omega$ & $N$ & $E(\Omega)$ & $R(\Omega)$ \\
\hline \multicolumn{4}{|c|}{$\left(\ell_{1}, \ell_{2}, \ell_{3}\right)=(0,0,1),(0,1,0)$ with $\chi_{1}$ only } \\
\hline 10 & 3024 & -7.41015653121966 & \\
\hline 11 & 4824 & -7.41015653231089 & \\
\hline 12 & 7440 & -7.41015653255834 & 4.409 \\
\hline 13 & 11118 & -7.41015653262575 & 3.670 \\
\hline 14 & 16164 & -7.41015653264083 & 4.470 \\
\hline 15 & 23004 & -7.41015653264843 & 1.983 \\
\hline 16 & 30224 & -7.41015653265066 & 3.402 \\
\hline \multicolumn{4}{|c|}{$\left(\ell_{1}, \ell_{2}, \ell_{3}\right)=(0,0,1),(0,1,0),(1,0,0)$ with $\chi_{1}$ only } \\
\hline 8 & 1016 & -7.410156502496189 & \\
\hline 9 & 1870 & -7.410156529965265 & \\
\hline 10 & 3300 & -7.410156532354927 & 11.49 \\
\hline 11 & 5600 & -7.410156532619679 & 9.026 \\
\hline 12 & 9170 & -7.410156532647379 & 9.557 \\
\hline 13 & 14532 & -7.410156532651514 & 6.698 \\
\hline 14 & 22400 & -7.410156532652175 & 6.257 \\
\hline 15 & 33600 & -7.410156532652360 & 3.575 \\
\hline$\infty$ & & $-7.4101565326524(1)$ & \\
\hline \multicolumn{4}{|c|}{$\left(\ell_{1}, \ell_{2}, \ell_{3}\right)=(0,0,1),(0,1,0),(1,0,0)$ with $\chi_{1}$ and $\chi_{2}$} \\
\hline 5 & 153 & -7.410148566764002 & \\
\hline 6 & 385 & -7.410155955712337 & \\
\hline 7 & 888 & -7.410156484860455 & 13.96 \\
\hline 8 & 1878 & -7.410156528148006 & 12.22 \\
\hline 9 & 3692 & -7.410156532067689 & 11.04 \\
\hline 10 & 6828 & -7.410156532624245 & 7.04 \\
\hline 11 & 11950 & -7.410156532650209 & 21.43 \\
\hline 12 & 20000 & -7.410156532652104 & 13.69 \\
\hline 13 & 32200 & -7.410156532652370 & 7.13 \\
\hline$\infty$ & & $-7.41015653265241(4)$ & \\
\hline \multicolumn{4}{|c|}{ Other calculations } \\
\hline 8 & 1715 & $-7.4101565184^{\mathrm{a}}$ & \\
\hline 12 & & $-7.4101565326286^{\mathrm{b}}$ & \\
\hline$\infty$ & & $-7.410156532665(14)^{\mathrm{b}}$ & \\
\hline
\end{tabular}

${ }^{\mathrm{a}}$ Yan et al. [2].

bPuchalski et al. [6].

The radial part of the fifth sector is generated by the formula

$$
j_{1}+j_{2}+j_{3}+j_{12}+j_{23}+j_{31} \leqslant \Omega-4,
$$

but terms with $j_{1}>j_{2}$ and $j_{1}=j_{2}$ when $j_{23}>j_{31}$ are omitted to avoid unnecessary duplication and potential linear dependence. The radial parts of the sixth sector and the seventh sector are also generated by formula (17), but no terms are omitted here. The first five sectors are assigned the angular momentum configuration $\left(\ell_{1}, \ell_{2}, \ell_{3}\right)=(0,0,1)$, and the sixth and seventh sectors are assigned configurations $\left(\ell_{1}, \ell_{2}, \ell_{3}\right)=(0,1,0)$ and $\left(\ell_{1}, \ell_{2}, \ell_{3}\right)=(1,0,0)$, respectively.

As can be seen from Table III, the energies decrease significantly when the third configuration is added, and the energy convergence ratios $R(\Omega)$ are much larger (i.e., faster convergence) when using three configurations than when using two configurations. $R(\Omega)$ is defined in terms of the ratio of successive differences by

$$
R(\Omega)=\frac{E(\Omega)-E(\Omega-1)}{E(\Omega-1)-E(\Omega-2)},
$$

and so it immediately reveals the pattern of convergence. The case $R=$ constant is the ideal case corresponding to geometric convergence. The results in Table III demonstrate that the third configuration $\left(\ell_{1}, \ell_{2}, \ell_{3}\right)=(1,0,0)$ is important for calculations of the $2 P$ state of lithium.

For the $3 D$ state of lithium, according to our analysis, there are in total six necessary angular momentum configurations. For completeness, we add these one by one and study their impact on the energy of the $3 D$ state, as listed in Table IV. It can be seen from the table that with only one angular momentum configuration $(0,0,2)$, the energy of the $1 s^{2} 3 d^{2} D$ state converges to -7.335523087 a.u., in which only the first seven figures are correct. When two configurations $(0,0,2)$ and $(0,1,1)$ are used, the variational energy decreases to -7.335523543078 , in which the first ten figures are correct. When three configurations $(0,0,2),(0,2,0)$, and $(0,1,1)$ are used, the variational energy with 25788 basis functions is -7.335523543523941 a.u., with almost thirteen correct figures. When four configurations $(0,0,2),(0,2,0),(2,0,0)$, and $(0,1,1)$ are used, the variational energy with 30000 basis functions is -7.335523543524386 a.u., which has thirteen correct figures. When five configurations $(0,0,2),(0,2,0)$, $(2,0,0),(0,1,1)$, and $(1,0,1)$ are used, the variational energy with 32760 basis functions is -7.335523543524685 a.u., which has fourteen or fifteen correct figures. It is significant that the $R(\Omega)$ convergence ratios are much larger with five configurations than they are for the other cases with fewer configurations. We find that adding the sixth and final configuration $(1,1,0)$ in Table II does not further lower the energy significantly, and so we do not list the results explicitly in Table IV.

\section{EFFECT OF THE SECOND SPIN FUNCTION ON ENERGIES AND SPIN-DEPENDENT MATRIX ELEMENTS}

As mentioned in the Introduction, there are two independent spin wave functions for the double states of lithium,

$$
\begin{aligned}
\chi_{1}(1,2,3)= & \alpha(1) \beta(2) \alpha(3)-\beta(1) \alpha(2) \alpha(3), \\
\chi_{2}(1,2,3)= & 2 \alpha(1) \alpha(2) \beta(3)-\beta(1) \alpha(2) \alpha(3) \\
& -\alpha(1) \beta(2) \alpha(3) .
\end{aligned}
$$

The physical meaning of $\chi_{1}$ is that electrons 1 and 2 are first coupled to a spin single state $S_{12}=0$, and the result coupled with electron 3 to give a final state with total spin $S=1 / 2$ and $z$ component $S_{z}=1 / 2 . \chi_{2}$ means that electrons 1 and 2 are first coupled to a spin triplet state $S_{12}=1$, and the result coupled with electron 3 to give a final state with total spin $S=1 / 2$ and $z$ component $S_{z}=1 / 2$. Larsson had given detailed discussions about the necessity of the second spin wave function in the calculations of the energy levels of lithium [13]. His conclusion is that if the basis set contains the terms generated by the operator

$$
\mathcal{A}\left\{(13) \phi \chi_{1}\right\}
$$


TABLE IV. Convergence study for the nonrelativistic energy of lithium in the $1 s^{2} 3 d^{2} D$ state, calculated using different angular momentum configurations. Units are atomic units.

\begin{tabular}{|c|c|c|c|}
\hline$\Omega$ & $N$ & $E(\Omega)$ & $R(\Omega)$ \\
\hline \multicolumn{4}{|c|}{$\left(\ell_{1}, \ell_{2}, \ell_{3}\right)=(0,0,2)$} \\
\hline 7 & 288 & -7.335522846909011 & \\
\hline 8 & 570 & -7.335523005011643 & \\
\hline 9 & 1050 & -7.335523078299127 & 2.157 \\
\hline 10 & 1830 & -7.335523086314969 & 9.142 \\
\hline 11 & 3040 & -7.335523087718336 & 5.711 \\
\hline \multicolumn{4}{|c|}{$\left(\ell_{1}, \ell_{2}, \ell_{3}\right)=(0,0,2),(0,1,1)$} \\
\hline 10 & 1785 & -7.335523540669860 & \\
\hline 11 & 3045 & -7.335523542483543 & \\
\hline 12 & 4970 & -7.335523542882444 & 4.471 \\
\hline 13 & 7820 & -7.335523543001790 & 3.342 \\
\hline 14 & 11952 & -7.335523543052819 & 2.338 \\
\hline 15 & 17780 & -7.335523543078357 & 2.018 \\
\hline \multicolumn{4}{|c|}{$\left(\ell_{1}, \ell_{2}, \ell_{3}\right)=(0,0,2),(0,1,1),(0,2,0)$} \\
\hline 9 & 1722 & -7.335523541259572 & \\
\hline 10 & 3180 & -7.335523543125368 & \\
\hline 11 & 5548 & -7.335523543444753 & 5.841 \\
\hline 12 & 9240 & -7.335523543510569 & 4.852 \\
\hline 13 & 13800 & -7.335523543520135 & 6.880 \\
\hline 14 & 16960 & -7.335523543522726 & 3.692 \\
\hline 15 & 25788 & -7.335523543523941 & 2.132 \\
\hline \multicolumn{4}{|c|}{$\left(\ell_{1}, \ell_{2}, \ell_{3}\right)=(0,0,2),(0,1,1),(0,2,0),(2,0,0)$} \\
\hline 8 & 704 & -7.335523524776523 & \\
\hline 9 & 1428 & -7.335523541000464 & \\
\hline 10 & 2700 & -7.335523543043150 & 7.942 \\
\hline 11 & 4888 & -7.335523543477152 & 4.706 \\
\hline 12 & 8400 & -7.335523543512381 & 12.31 \\
\hline 13 & 13832 & -7.335523543522043 & 3.646 \\
\hline 14 & 21966 & -7.335523543524045 & 4.824 \\
\hline 15 & 30000 & -7.335523543524386 & 5.876 \\
\hline \multicolumn{4}{|c|}{$\left(\ell_{1}, \ell_{2}, \ell_{3}\right)=(0,0,2),(0,1,1),(0,2,0),(2,0,0),(1,0,1)$} \\
\hline 8 & 780 & -7.335523524256807 & \\
\hline 9 & 1638 & -7.335523541397026 & \\
\hline 10 & 3180 & -7.335523543374304 & 8.688 \\
\hline 11 & 5810 & -7.335523543510671 & 14.49 \\
\hline 12 & 10115 & -7.335523543523117 & 10.95 \\
\hline 13 & 16835 & -7.335523543524362 & 9.997 \\
\hline 14 & 26955 & -7.335523543524655 & 4.177 \\
\hline 15 & 32760 & -7.335523543524685 & 9.820 \\
\hline$\infty$ & \multicolumn{3}{|c|}{$-7.335523543524688(3)$} \\
\hline \multicolumn{4}{|c|}{ Other calculations } \\
\hline 8 & 1673 & $-7.33552354035^{\mathrm{a}}$ & \\
\hline \multirow[t]{2}{*}{$\infty$} & & $-7.33552354110(43)^{\mathrm{a}}$ & \\
\hline & 4000 & $-7.33552354297^{\mathrm{b}}$ & \\
\hline
\end{tabular}

a'Yan et al. [2].

${ }^{\mathrm{b}}$ Sharkey et al. [25].

then, according to this argument, the second spin function need not be explicitly included. Here, $\mathcal{A}$ is the three-particle antisymmetrizer and (13) denotes the exchange of electron 1 and electron 3 operating on $\phi$ (the orbital part of the wave function). The energy lowering when $\mathcal{A}\left\{\phi \chi_{2}\right\}=\mathcal{A}\left\{(13) \phi \chi_{1}\right\}$ is included would then be small.
In the following two sections, we present extensive numerical tests of the effect of the second spin function on energies and expectation values of spin-dependent operators, such as the Fermi contact term.

\section{A. Second spin function and energies}

In Table $\mathrm{V}$, we list the energies for the ground state of lithium calculated using only $\chi_{1}$, only $\chi_{2}$, and both $\chi_{1}$ and $\chi_{2}$, respectively. The energies calculated using only $\chi_{1}$ are cited from our recent paper [9]. The radial parts of the basis set for using $\chi_{2}$ are obtained by exchanging $j_{1}$ with $j_{3}$ and $j_{12}$ with $j_{23}$ of the radial parts of the basis set for using $\chi_{1}$. The radial part of the basis set for using both $\chi_{1}$ and $\chi_{2}$ is divided into six sectors. The first four sectors are constructed according to the basis manner described in Sec. II, namely, formula (8). The fifth sector is generated by the following manner:

$$
j_{1}+j_{2}+j_{3}+j_{12}+j_{23}+j_{31} \leqslant \Omega-3,
$$

but terms like $j_{1}>j_{2}$ and $j_{1}=j_{2}$ when $j_{23}>j_{31}$ are omitted for avoiding the potential linear dependence. The sixth sector is also generated by Eq. (22), but when $\Omega<10$ the terms that should be omitted are the same as the fifth sector; when $\Omega \geqslant 10$, terms like $j_{1}>j_{3}$ and $j_{12}>j_{31}$ when $j_{1}=j_{3}$ are omitted.

It can be seen from Table $\mathrm{V}$ that the energy of the ground state of the lithium can be calculated to -7.478060323910134 a.u. with 26520 basis functions only using $\chi_{1}$, and it can be calculated to -7.478060323909942 a.u. with 26520 basis functions only using $\chi_{2}$. When both $\chi_{1}$ and $\chi_{2}$ are used, the energy can be calculated to -7.478060323910146894 a.u. with 34020 basis functions. Comparing these results, we can make the conclusion that the nonrelativistic energy levels of lithium do not depend on the spin wave functions. No matter whether $\chi_{1}$ or $\chi_{2}$ or both $\chi_{1}$ and $\chi_{2}$ are used, the energy of the ground state of the lithium converges to the same limit. However, if both $\chi_{1}$ and $\chi_{2}$ are used, the energy converges slightly more quickly. In Tables VI and VII, we list the energies of $3 S-6 S$ states of lithium which are calculated using $\chi_{1}$ and both $\chi_{1}$ and $\chi_{2}$, respectively. Here also, adding the second spin wave function slightly improves the convergence ratios.

We also explored the effect of the second spin function for the $2 P$ state, using a basis set divided into ten sectors. The first seven sectors were the same as those in the one-spin case, and with the same $\Omega$ for the highest sum of powers. The last three sectors (i.e., 8, 9, and 10) were assigned the second spin function and angular momentum configurations $(0,0,1)$, $(0,1,0)$, and $(1,0,0)$, respectively, but with the smaller sum of powers $\Omega_{2}=\Omega-3$ [cf. Eq. (22)]. In addition, the terms in the eighth sector were truncated such that terms with $j_{1} \geqslant j_{2}$ when $j_{23}>j_{31}$ were omitted. The results are included in Table III. As can be seen, the pattern of convergence is unusually smooth for this case, and yields the best variational bound for the $2 P$ state. The results with and without the second spin function agree with each other within the estimated uncertainty.

\section{B. Second spin function and the Fermi contact term}

For expectation values of spin-dependent operators, such as the Fermi contact term, one might argue that the effect of 
TABLE V. Convergence study for the nonrelativistic energy of Li in the ground state, calculated using only the first spin wave function $\chi_{1}$, the second spin wave function $\chi_{2}$, and both spin wave functions $\chi_{1}$ and $\chi_{2}$, respectively. $N$ is the number of terms, and $\Omega=\infty$ denotes the extrapolated value. Units are atomic units.

\begin{tabular}{|c|c|c|c|}
\hline$\Omega$ & $N$ & $E(\Omega)$ & $R(\Omega)$ \\
\hline \multicolumn{4}{|c|}{ With only $\chi_{1}$} \\
\hline 10 & 3910 & -7.478060323880889 & \\
\hline 11 & 6039 & -7.478060323905362 & \\
\hline 12 & 9056 & -7.478060323909450 & 5.986 \\
\hline 13 & 13248 & -7.478060323909950 & 8.174 \\
\hline 14 & 18935 & -7.478060323910102 & 3.290 \\
\hline 15 & 26520 & -7.478060323910134 & 4.679 \\
\hline \multicolumn{4}{|c|}{ With only $\chi_{2}$} \\
\hline 10 & 3910 & -7.478060323491870 & \\
\hline 11 & 6039 & -7.478060323825035 & \\
\hline 12 & 9056 & -7.478060323891747 & 4.994 \\
\hline 13 & 13248 & -7.478060323902848 & 6.009 \\
\hline 14 & 18935 & -7.478060323908907 & 1.832 \\
\hline 15 & 26520 & -7.478060323909791 & 6.851 \\
\hline \multicolumn{4}{|c|}{ With both $\chi_{1}$ and $\chi_{2}$} \\
\hline 10 & 5082 & -7.478060323905585516 & \\
\hline 11 & 7992 & -7.478060323909524819 & \\
\hline 12 & 12168 & -7.478060323910044374 & 7.582 \\
\hline 13 & 18108 & -7.478060323910127997 & 6.213 \\
\hline 14 & 24552 & -7.478060323910144868 & 4.956 \\
\hline 15 & 34020 & -7.478060323910146894 & 8.327 \\
\hline$\infty$ & & $-7.478060323910147(1)$ & \\
\hline Sims et al. $[10]$ & 16764 & -7.478060323452 & \\
\hline Stanke et al. [11] & 10000 & -7.47806032381 & \\
\hline Yan et al. [4] & 9577 & -7.4780603238924 & \\
\hline Puchalski et al. [8] & 30632 & -7.478060323910097 & \\
\hline Puchalski et al. [8] & $\infty$ & $-7.4780603239102(2)$ & \\
\hline
\end{tabular}

TABLE VI. Convergence study for the nonrelativistic energies of Li in the $1 s^{2} 3 s^{2} S$ and $1 s^{2} 4 s^{2} S$ states, calculated using the spin wave function $\chi_{1}$ and the spin wave functions $\chi_{1}$ and $\chi_{2}$, respectively. $N$ is the number of terms, and $\Omega=\infty$ denotes the extrapolated value. Units are atomic units.

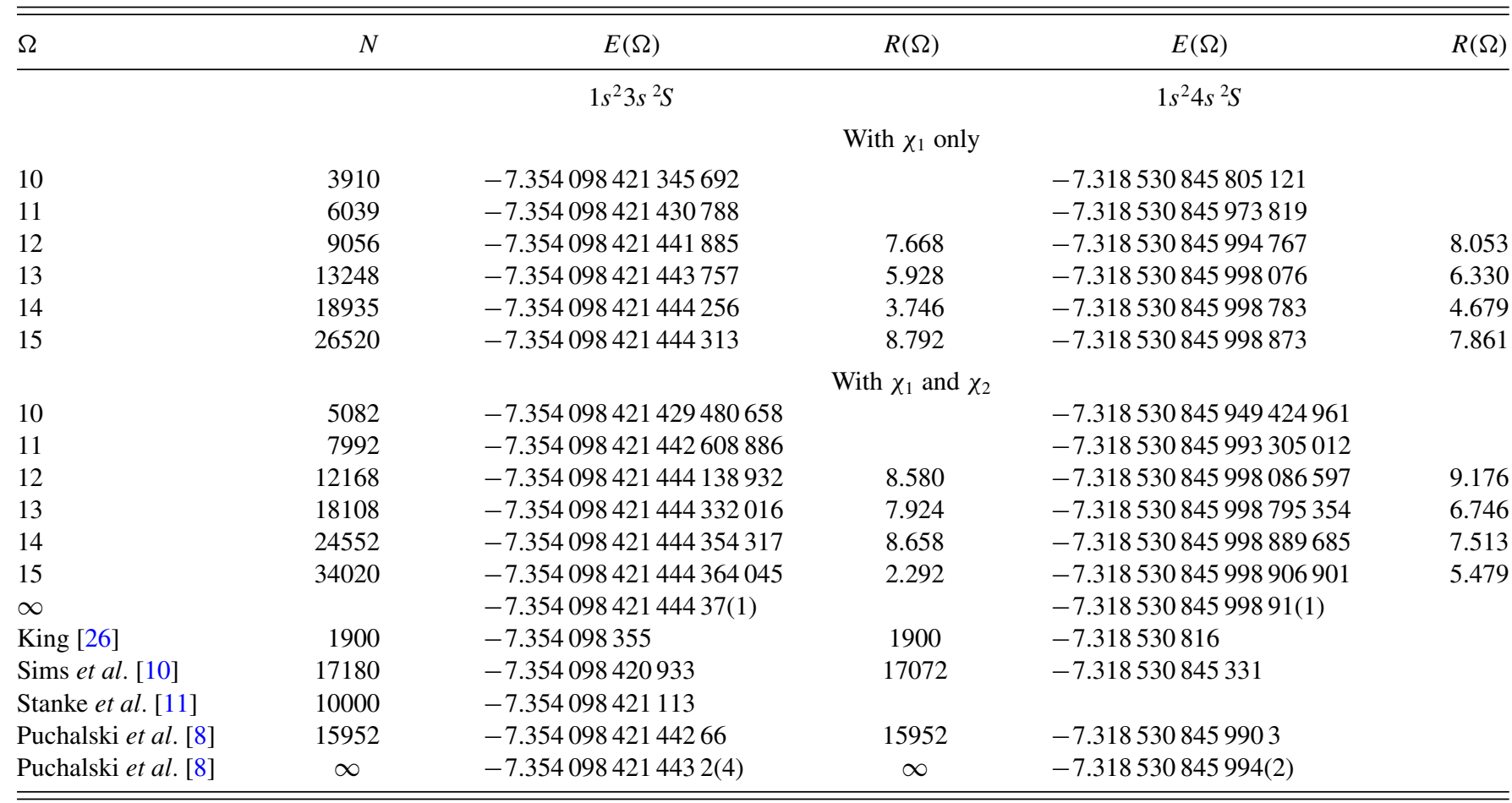


TABLE VII. Convergence study for the nonrelativistic energies of Li in the $1 s^{2} 5 s^{2} S$ and $1 s^{2} 6 s{ }^{2} S$ state, calculated using the spin wave function $\chi_{1}$ and the spin wave functions $\chi_{1}$ and $\chi_{2}$, respectively. $N$ is the number of terms and $\Omega=\infty$ denotes the extrapolated value. Units are atomic units.

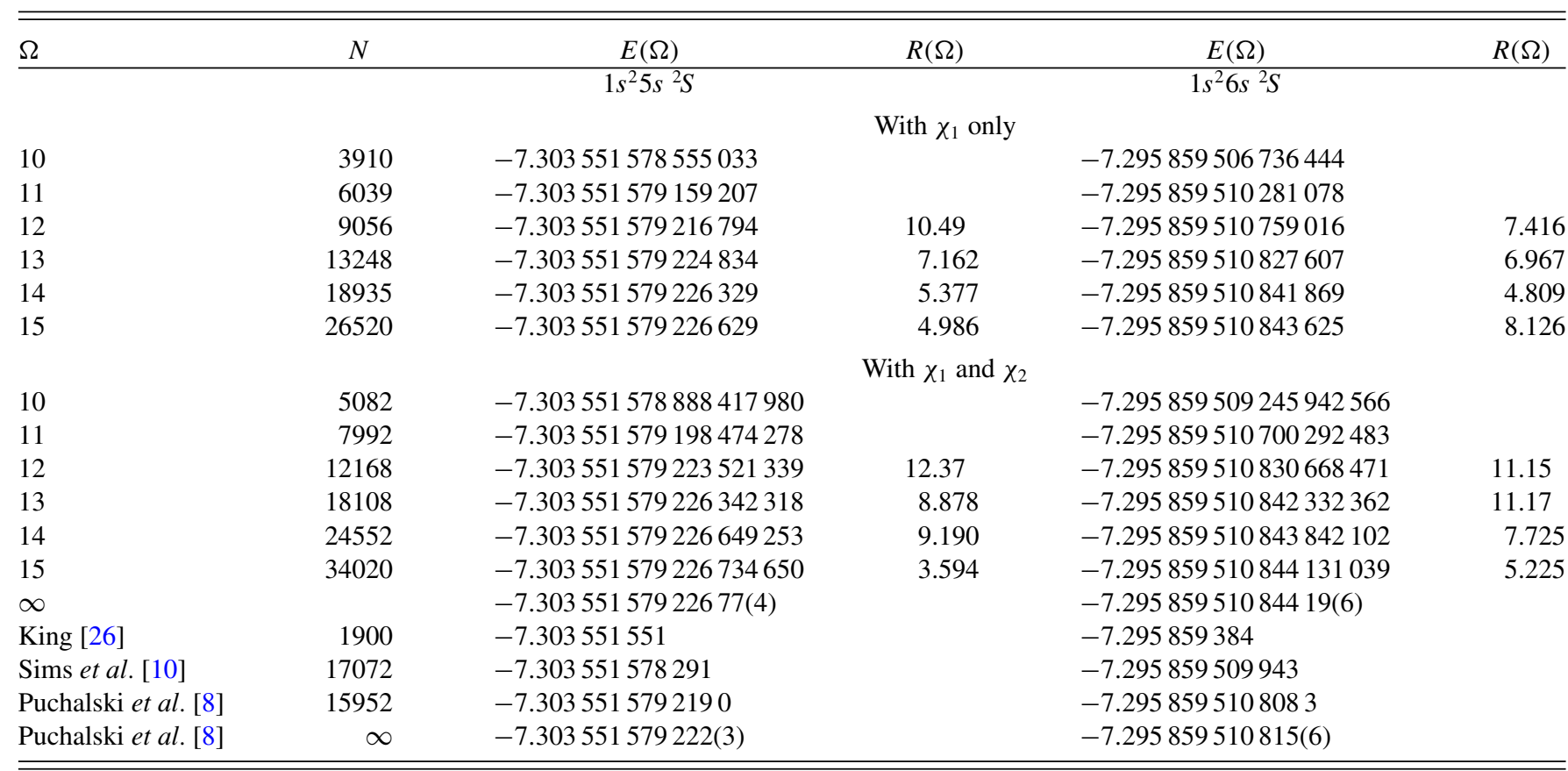

the second spin wave function may be more important. The circumstantial evidence presented by King $[16,17]$ seemed to indicate that the rate of convergence was greatly accelerated by adding the second spin function, based on basis sets with 332 terms vs 602 terms with both spin functions.

In order to settle the question, we have carried out extensive calculations using only the first spin function, and both spin functions together. The uncorrected Fermi contact factor $f_{\mathrm{c}}$ is defined by

$$
f_{\mathrm{c}}=4 \pi\left\langle\Psi\left|\sum_{i=1}^{3} \delta\left(\mathbf{r}_{i}\right) \sigma_{z i}\right| \Psi\right\rangle,
$$

where $\hbar \sigma_{z i} / 2$ is the spin operator of electron $i$ in the $z$ direction, and $\Psi$ is the nonrelativistic wave function of lithium for infinite nuclear mass. This is related to the full hyperfine structure coupling constant $A_{1 / 2}(\exp )$ by

$$
\begin{aligned}
A_{1 / 2}(\exp )= & 2\left(1+a_{e}\right) C_{\mathrm{rel}} C_{M} C_{R} C_{\mathrm{QED}} \\
& \times 95.41067(7) \frac{\mu_{I}}{3 I} f_{\mathrm{c}} \mathrm{MHz},
\end{aligned}
$$

where $\mu_{I}$ is the magnetic moment of the nucleus, $a_{e}$ is the anomalous magnetic moment correction, $C_{\text {rel }}$ is the relativistic correction factor, $C_{M}$ and $C_{R}$ are the finite nuclear mass and size correction factors, and $C_{\mathrm{QED}}$ is the QED correction factor other than the anomalous magnetic moment correction (see Ref. [27] for further details).

The results of calculations of $f_{\mathrm{c}}$ for the $2 S$ and $3 S$ states of lithium are shown in Table VIII. The ratios $R(\Omega)$ of successive differences indicate that the convergence is generally erratic, but the differences between successive calculations nevertheless progressively decrease. The significant point is that the number of figures that are correct are about the same for basis sets of approximately the same size. The final result is more accurate with two spin functions only because the total basis set size is larger. The final result for the $2 S$ state of $f_{\mathrm{c}}=2.90596895(5)$ a.u. is in harmony with our previous value $2.905922(50)$, and with the effective nonrelativistic result 2.90589 extracted from the large-scale relativistic CI calculations of Yerokhin [29]. It seems clear that the larger value $2.90856(8)$ a.u. obtained by Esquival et al. is overestimated. The results in Table VIII for the $3 S$ state are in similarly good agreement with our previous calculations and those of Yerokhin [29]. The value with two spin functions has evidently converged to the very accurate result $f_{\mathrm{c}}=0.673380816(3)$ a.u. Previous experience with high precision Hylleraas calculations for helium [30] indicates that the accuracy for both states could be further improved by a factor of 20 or more by use of the Hiller-SucherFeinberg (HSF) [31] global operator. For wave functions of low accuracy, or for Gaussian basis sets [32], even larger improvements in accuracy have been obtained with both the HSF and Drachman [33] global operators.

Results for the higher-lying $S$ states, the $2 P$ state, and the $3 D$ state are summarized in Table IX. For most cases, the results with one spin function and both spin functions agree within the estimated uncertainties, although the $2 P$ state appears to be anomalous in this regard, undoubtedly due to the erratic nature of the convergence.

The conclusion from all the results taken together is that the second spin function has no direct effect on the final converged value of expectation values, even for spin-dependent operators such as $f_{\mathrm{c}}$. However, the rate of convergence may depend on the strategy used to construct the variational wave function. The multiplicity of distance scales used in the present work for the spatial part appears to produce the same enhancement of convergence as including the second spin function explicitly, 
TABLE VIII. Fermi contact term $f_{\mathrm{c}}$ for the Li $2 S$ and $3 S$ states, with and without the second spin function $\chi_{2} . N$ is the number of terms and $\Omega=\infty$ denotes the extrapolated value. Units are atomic units.

\begin{tabular}{|c|c|c|c|c|c|}
\hline$\Omega$ & $N$ & $f_{\mathrm{c}}$ & $R(\Omega)$ & $f_{\mathrm{c}}$ & $R(\Omega)$ \\
\hline \multicolumn{4}{|c|}{$1 s^{2} 2 s^{2} S$} & \multicolumn{2}{|l|}{$1 s^{2} 3 s^{2} S$} \\
\hline \multicolumn{6}{|c|}{ With $\chi_{1}$ only } \\
\hline 5 & 255 & 2.909653645056 & & 0.680191820971 & \\
\hline 6 & 500 & 2.906774702366 & & 0.674102759251 & \\
\hline 7 & 910 & 2.905845558005 & 3.098 & 0.673273729239 & 7.344 \\
\hline 8 & 1580 & 2.906015663946 & -5.462 & 0.673414603634 & -5.884 \\
\hline 9 & 2620 & 2.905998447250 & -9.880 & 0.673412297822 & -61.099 \\
\hline 10 & 3910 & 2.905952999341 & 0.378 & 0.673384394853 & 0.083 \\
\hline 11 & 6039 & 2.905970621969 & -2.578 & 0.673377420547 & 4.000 \\
\hline 12 & 9056 & 2.905969401324 & -14.43 & 0.673381377367 & -1.762 \\
\hline 13 & 13248 & 2.905969443355 & -29.04 & 0.673380631643 & -5.306 \\
\hline 14 & 18935 & 2.905968909939 & -7.879 & 0.673380926602 & -2.528 \\
\hline 15 & 26520 & 2.905968946277 & -14.67 & 0.673380729330 & -5.678 \\
\hline$\infty$ & & $2.905968944(37)$ & & $0.67338081(19)$ & \\
\hline \multicolumn{6}{|c|}{ With $\chi_{1}$ and $\chi_{2}$} \\
\hline 5 & 270 & 2.901676269089 & & 0.676996860796 & \\
\hline 6 & 550 & 2.905038297856 & & 0.672848734456 & \\
\hline 7 & 1024 & 2.906005450054 & 3.476 & 0.673624710626 & -5.345 \\
\hline 8 & 1840 & 2.905974291454 & -31.03 & 0.673327460084 & -2.610 \\
\hline 9 & 3125 & 2.905987258807 & -2.402 & 0.673386386061 & -5.044 \\
\hline 10 & 5082 & 2.905968903909 & -0.706 & 0.673382187111 & -6.988 \\
\hline 11 & 7992 & 2.905969903592 & -18.36 & 0.673381678637 & -2.263 \\
\hline 12 & 12618 & 2.905968826597 & -0.928 & 0.673380436264 & -3.008 \\
\hline 13 & 18188 & 2.905968925663 & -10.87 & 0.673380904796 & -2.643 \\
\hline 14 & 26250 & 2.905969012972 & 1.135 & 0.673380813175 & -4.135 \\
\hline 15 & 34020 & 2.905968915597 & -0.897 & 0.673380816397 & -5.433 \\
\hline$\infty$ & & $2.905968967(52)$ & & $0.673380816(3)$ & \\
\hline Yan et al. [27] & & $2.905922(50)$ & & $0.67341(5)$ & \\
\hline Esquivel et al. [28] & & $2.90856(8)$ & & & \\
\hline Yerokhin [29] & & 2.90589 & & 0.67336 & \\
\hline
\end{tabular}

as observed by King [16,17]. This conclusion applies only to first-order perturbation corrections. The second spin function would play a crucial role in sums over virtual intermediate states for higher-order perturbation corrections due to spindependent operators.

\section{SUMMARY}

In this paper, we discussed the completeness of the Hylleraas-type basis set for three-electron atomic systems, with the $2 P$ and $3 D$ states as examples to demonstrate the impact of the various angular moment configurations to the energy levels. We presented the most accurate nonrelativistic

TABLE IX. Fermi contact term $f_{\mathrm{c}}$ for excited states of lithium, with and without the second spin function. Units are atomic units.

\begin{tabular}{lcc}
\hline \hline State & With $\chi_{1}$ only & With $\chi_{1}$ and $\chi_{2}$ \\
\hline $4 S$ & $0.25397348(2)$ & $0.25397358(1)$ \\
$5 S$ & $0.1218672(5)$ & $0.12186722(1)$ \\
$6 S$ & $0.0675946(1)$ & $0.0675944(2)$ \\
$2 P$ & $-0.21461783(6)$ & $-0.21461775(2)$ \\
$3 D$ & $-0.00035439(5)$ & \\
\hline
\end{tabular}

energies and wave functions available for the $2 S-6 S, 2 P$, and $3 D$ states of lithium. It is well established in the literature that the energies are not sensitive to the second spin wave function, and that is fully confirmed in this work. What is not so well established is the role of the second spin function for spin-dependent operators. Contrary to previous work [16,17], our results show that the second spin function does not affect diagonal expectation values, such as the Fermi contact term, provided that there is sufficient flexibility in the spatial part of the variational wave function. The accuracy of this term is substantially improved for the $2 S-6 S, 2 P$, and $3 D$ states of lithium. These results lay the foundation for improved calculations of corrections due to relativistic and QED effects, and applications to isotope shifts [4].

\section{ACKNOWLEDGMENTS}

This work was supported by NNSF of China under Grant No. 10874133, by NSERC of Canada, and by the Canadian computing facilities of SHARCnet and ACEnet. We thank Fuqing Liu very much for his valuable advice. The computer facilities (No. TC5000) at the School of Physics, Wuhan University are gratefully acknowledged. 
[1] D. K. McKenzie and G. W. F. Drake, Phys. Rev. A 44, R6973 (1991).

[2] Z.-C. Yan and G. W. F. Drake, Phys. Rev. A 52, 3711 (1995).

[3] Z.-C. Yan, M. Tambasco, and G. W. F. Drake, Phys. Rev. A 57, 1652 (1998).

[4] Z.-C. Yan, W. Nörtershäuser, and G. W. F. Drake, Phys. Rev. Lett. 100, 243002 (2008).

[5] M. Puchalski and K. Pachucki, Phys. Rev. A 73, 022503 (2006).

[6] M. Puchalski and K. Pachucki, Phys. Rev. A 78, 052511 (2008).

[7] M. Puchalski, D. Kedziera, and K. Pachucki, Phys. Rev. A 80, 032521 (2009).

[8] M. Puchalski, D. Kedziera, and K. Pachucki, Phys. Rev. A 82, 062509 (2010).

[9] L. M. Wang, Z.-C. Yan, H. X. Qiao, and G. W. F. Drake, Phys. Rev. A 83, 034503 (2011).

[10] J. S. Sims and S. A. Hagstrom, Phys. Rev. A 80, 052507 (2009).

[11] M. Stanke, J. Komasa, D. Kedziera, S. Bubin, and L. Adamowicz, Phys. Rev. A 78, 052507 (2008).

[12] K. Singer, Proc. Phys. Soc., London, Sect. A 258, 412 (1960).

[13] S. Larsson, Phys. Rev. 169, 49 (1968).

[14] U. Kaldor and F. E. Harris, Phys. Rev. 183, 1 (1969).

[15] A. Lüchow and H. Kleindienst, Int. J. Quantum Chem. 51, 211 (1994).

[16] F. W. King and V. Shoup, Phys. Rev. A 33, 2940 (1986).

[17] F. W. King, Phys. Rev. A 40, 1735 (1989).

[18] G. Breit, Phys. Rev. 35, 569 (1930).

[19] C. Schwartz, Phys. Rev. 123, 1700 (1961).
[20] G. W. F. Drake, in Long-Range Casimir Forces: Theory and Recent Experiments on Atomic Systems, edited by F. S. Levin and D. A. Micha (Plenum, New York, 1993).

[21] F. E. Harris, Adv. Quantum Chem. 50, 61 (2005).

[22] B. Klahn and W. A. Bingel, Theor. Chim. Acta (Berl) 44, 9 (1977); 44, 27 (1977); Int. J. Quantum Chem. 11, 943 (1977).

[23] G. W. F. Drake, in Mathematical Tools for Physicists, edited by George L. Trigg (Wiley-VCH Verlag GmbH \& Co. KGaA, Weinheim, 2005), pp. 619-656.

[24] A. R. Edmonds, Angular Momentum in Quantum Mechanics (Princeton University Press, Princeton, NJ, 1957).

[25] K. L. Sharkey, S. Bubin, and L. Adamowicz, Phys. Rev. A 83, 012506 (2011).

[26] F. W. King, Phys. Rev. A 76, 042512 (2007).

[27] Z.-C. Yan, D. K. McKenzie, and G. W. F. Drake, Phys. Rev. A 54, 1322 (1996).

[28] R. O. Esquivel, A. Vivier-Bunge, and V. H. Smith Jr., J. Mol. Struct.: THEOCHEM 433, 43 (1998).

[29] V. A. Yerokhin, Phys. Rev. A 78, 012513 (2008).

[30] G. W. F. Drake, Phys. Rev. A 45, 70 (1992) [see text following Eq. (88)]; G. W. F. Drake and Z.-C. Yan, ibid. 46, 2378 (1992), Table VI.

[31] J. Hiller, J. Sucher, and G. Feinberg, Phys. Rev. A 18, 2399 (1978); J. Hiller, J. Sucher, A. K. Bhatia, and G. Feinberg, ibid. 21, 1082 (1980).

[32] K. Pachucki, W. Cencek, and J. Komasa, J. Chem. Phys. 122, 184101 (2005).

[33] R. Drachman, J. Phys. B 14, 2733 (1981). 